\title{
Article \\ Clonal Hematopoiesis after Autologous Stem Cell Transplantation Does Not Confer Adverse Prognosis in Patients with AML
}

\author{
Alexander D. Heini ${ }^{1,+}$, Naomi Porret ${ }^{2,+}$, Reinhard Zenhaeusern ${ }^{3}$, Annette Winkler ${ }^{4}$, Ulrike Bacher ${ }^{2}$ (D) \\ and Thomas Pabst ${ }^{1, *}$
}

1 Department of Medical Oncology, University Hospital and University of Bern, Center for Hemato-Oncology, University Cancer Center, 3010 Berne, Switzerland; alexander.heini@insel.ch

2 Department of Hematology and Central Hematology Laboratory, Inselspital, Bern University Hospital, University of Bern, 3010 Berne, Switzerland; NaomiAzur.Porret@insel.ch (N.P.); veraulrike.bacher@insel.ch (U.B.)

3 Department of Medical Oncology, Spitalzentrum Oberwallis, 3900 Brig, Switzerland; reinhard.zenhaeusern@hopitalvs.ch

4 Regionalspital Biel, 2501 Biel, Switzerland; anette.winkler@szb-chb.ch

* Correspondence: thomas.pabst@insel.ch

+ A.D.H. and N.P. contributed equally.

check for updates

Citation: Heini, A.D.; Porret, N.; Zenhaeusern, R.; Winkler, A.; Bacher, U.; Pabst, T. Clonal Hematopoiesis after Autologous Stem Cell Transplantation Does Not Confer Adverse Prognosis in Patients with AML. Cancers 2021, 13, 3190. https:// doi.org/10.3390/cancers13133190

Academic Editors: Riccardo Masetti and Luisa Strocchio

Received: 23 May 2021

Accepted: 22 June 2021

Published: 25 June 2021

Publisher's Note: MDPI stays neutral with regard to jurisdictional claims in published maps and institutional affiliations.

Copyright: (c) 2021 by the authors. Licensee MDPI, Basel, Switzerland. This article is an open access article distributed under the terms and conditions of the Creative Commons Attribution (CC BY) license (https:// creativecommons.org/licenses/by/ $4.0 /)$.
Simple Summary: Around 50\% of patients with acute myeloid leukemia (AML) achieve a definite cure with intensive chemotherapy and consolidation, but relapse remains the main cause of death. Clonal hematopoiesis $(\mathrm{CH})$ describes the presence of a clonal subset of myeloid precursors without known hematologic disease. We aimed to investigate whether the presence of $\mathrm{CH}$-related mutations in the three most common genes (DNMT3A, TET2, and ASXL1, called DTA mutations) after autologous stem cell transplantation (ASCT) influence the outcome and retrospectively analyzed samples of 110 AML patients. We found no significant impact from the presence of DTA-CH on progression-free or overall survival. Thus, the persistence of DTA mutations after induction treatment should not prevent AML patients in first remission from ASCT consolidation. These results should undergo verification in independent cohorts.

Abstract: Introduction: Despite a 50\% cure rate, relapse remains the main cause of death in patients with acute myeloid leukemia (AML) consolidated with autologous stem cell transplantation (ASCT) in first remission (CR1). Clonal hematopoiesis of indeterminate potential $(\mathrm{CH})$ increases the risk for hematological and cardiovascular disorders and death. The impact of $\mathrm{CH}$ persisting after ASCT in AML patients is unclear. Materials and Methods: We retrospectively investigated the prognostic value of persisting DNMT3A, TET2, or ASXL1 (DTA) mutations after ASCT. Patients underwent stratification depending on the presence of DTA mutations. Results: We investigated 110 consecutive AML patients receiving ASCT in CR1 after two induction cycles at our center between 2007 and 2020. $\mathrm{CH}$-related mutations were present in 31 patients (28.2\%) after ASCT. The baseline characteristics were similar between patients with or without persisting DTA mutations after ASCT. The median progression free survival was 26.9 months in patients without DTA mutations and 16.7 months in patients with DTA mutations (HR $0.75(0.42-1.33), p=0.287)$, and the median overall survival was 80.9 and 54.4 months (HR $0.79(0.41-1.51), p=0.440)$, respectively. Conclusion: We suggest that DTA-CH after ASCT is not associated with an increased risk of relapse or death. The persistence of DTA mutations after induction should not prevent AML patients in CR1 from ASCT consolidation. Independent studies should confirm these data.

Keywords: acute myeloid leukemia (AML); autologous stem cell transplantation (ASCT); clonal hematopoiesis $(\mathrm{CH})$; prognosis; outcome 


\section{Introduction}

Patients with acute myeloid leukemia (AML) considered fit for intensive treatment are treated with one or two cycles of intensive induction chemotherapy, followed by consolidation with either additional chemotherapy cycles along with autologous or allogeneic hematopoietic stem cell transplantation. The stratification of patients for these consolidation treatment options relies on the genetic risk assessed by cytogenetics and molecular analyses at initial diagnosis, the response to induction treatment, and the morbidity and mortality associated with the treatment options $[1,2]$. The concept of minimal or measurable residual disease (MRD) after induction has a significant impact on the type of consolidation and, increasingly, maintenance treatment [3]. MRD is defined as the detection of malignant cells at a submicroscopic level, and it has been shown to be an independent prognostic factor for the risk of relapse and death [4]. Multiparameter flow cytometry as well as molecular methods are routinely used for diagnosis and screening for residual disease in AML patients. While recurrent mutations in genes such as FLT3 or NPM1 have been incorporated in risk assessment for a long time [1,3], the introduction of next-generation sequencing (NGS) into the hematologic routine has enabled the discovery of a multitude of additional and often novel mutations [5]. The detection of druggable mutations has also opened the door for customized treatment strategies, some of which were integrated in the European LeukemiaNet (ELN) risk assessment and treatment for patients with AML $[1,6,7]$.

With increasing age, somatic mutations accumulate, owing to radiation, smoking, drug exposition, or impaired DNA repair mechanisms. It is estimated that around 10-15\% of individuals aged around 70 and $20 \%$ of those aged around 90 have clonal hematopoiesis, which accounts for $\geq 10 \%$ of circulating nucleated cells [8]. This clonal hematopoiesis $(\mathrm{CH})$ is defined as the presence of a somatic mutation associated with hematological malignancies such as DNMT3A, TET2, or ASXL1 (DTA mutations) without morphologic or clinical evidence of a hematological neoplasm, similar to the monoclonal gammopathy of undetermined significance (MGUS) [9] or monoclonal B-cell lymphocytosis (MBL). The odds of progression of $\mathrm{CH}$ to an overt myeloid neoplasia are $0.5-1 \%$ per year [10]. Importantly, patients with $\mathrm{CH}$ were identified to be at a higher risk for the development of not only hematological malignancies, but also inflammation or vascular diseases and adverse survival outcomes [11,12].

Up to $50 \%$ of AML patients consolidated, after intensive chemotherapy induction, in first remission with high-dose chemotherapy and autologous stem cell transplantation (ASCT) achieve a definitive cure. Despite this, relapse remains the major cause of mortality in these patients, and outcomes vary widely between different subsets of patients [13-15]. While the presence of recurrent leukemia-associated mutations was shown to have an adverse prognostic impact in patients with AML undergoing intensive treatment, this correlation has yet to be explored for $\mathrm{CH}$ related mutations. Considering these facts, a more in-depth analysis assessing the role of $\mathrm{CH}$ in AML patients after autologous transplantation is still missing and warranted.

\section{Materials and Methods}

\subsection{Study Design and Patients}

This retrospective single-center analysis included consecutive patients with AML undergoing high-dose chemotherapy (HDCT) with autologous stem cell transplantation (ASCT) between February 2007 and February 2020 at the University Hospital of Bern in Switzerland. De novo and secondary AML cases were included. Data were collected for demographic details, peripheral blood values, bone marrow and peripheral blood cytomorphology, molecular and cytogenetic abnormalities, and marrow and blood assessments during the follow-up while under and following therapy. The primary study end points were progression-free and overall survival. 


\subsection{Treatment}

Patients were treated within or according to the following protocols of the HOVON (a Dutch-Belgian hemato-oncology group) and the Swiss Group for Clinical Cancer Research (SAKK) AML groups: SAKK/HOVON-42, -81, -92, -102, and -132. Induction treatment consisted of one or two cycles of cytarabine and an anthracycline (either idarubicin or daunorubicin). Consolidation treatment consisted of HDCT with melphalan and cyclophosphamide or treosulfan, followed by autologous stem cell transplantation. Patients had to have an Eastern Cooperative Oncology Group performance status of 0-2 and no previous treatment for AML, with the exception of hydroxyurea for initial cytoreduction. The stem cells were harvested from peripheral blood after G-CSF stimulation in all cases.

\subsection{Risk and Response Assessment}

Genetic and molecular biologic risk profiling were applied according to the European LeukemiaNet (ELN) update from 2017 [1]. The response was assessed according to international working group (ELN) criteria. Morphologic complete remission (CR) was defined as bone marrow blasts of $<5 \%$ with complete hematologic recovery (absolute neutrophil count (ANC) $\geq 1.0 \mathrm{G} / \mathrm{L}$, platelets $\geq 100 \mathrm{G} / \mathrm{L}$, and no transfusion dependence) [16].

\subsection{Survival}

Progression-free survival (PFS) was defined as the time from ASCT to disease progression or death from any cause or the last follow-up, whichever occurred first. Overall survival (OS) was defined as the time between ASCT and death from any cause or until the last follow-up. Subjects lost to the follow-up were censored at the time last known to be alive.

\subsection{Molecular Analyses}

Molecular analyses were performed during first complete remission as described above. Samples were taken 113 days after ASCT in the median (93 days, ranging from 154 days before to 5793 days after ASCT in the $\mathrm{CH}$ group and 134 days, ranging from 181 days before to 3524 days after ASCT in the no $\mathrm{CH}$ group). For patients lost to the follow-up or death, analyses were performed from cryoconserved material stored upon ASCT. Of the samples, $60.0 \%$ were derived from peripheral blood, $37.2 \%$ were from bone marrow, and $2.8 \%$ were analyzed in the stem cell product after apheresis. Next-generation sequencing (NGS) was performed by a ThermoFisher Ion Torrent S5. Analyses included ASXL1 exons 11-12 (fragment length analysis for exon 12), DNMT3A exons 11-23, and TET2 (whole gene).

\subsection{Assessment and Definition of Clonal Hematopoiesis}

Molecular mutation assessments were performed at the initial diagnosis and during a follow-up. CH was defined as the persistence of mutations in DNMT3A, TET2, or ASXL1 at a variant allele frequency (VAF) level of at least $2 \%$ during complete remission [8]. Different cutoffs ( $5 \%$ and $10 \%$ ) were also tested. DTA mutations that were cleared after intensive chemotherapy and ASCT were considered leukemia-related, whereas persisting DTA mutations with VAF levels of $\geq 2 \%$, despite achieving hematologic $C R$ and despite of the clearance of other coincidental mutations at a follow-up, were considered to be $\mathrm{CH}$.

\subsection{Statistical Analyses}

All reported $p$-values were from two-tailed Fisher's or unpaired $t$-tests. A value of $p<0.05$ was considered statistically significant. Survival analyses were performed using the log-rank method. Analyses for the median follow-up were performed using reverse Kaplan-Meier estimates; thus, no $p$ value was reported. Statistical analyses were performed in GraphPad Prism Version 8 (GraphPad Software, Inc., LaJolla, CA, USA). 


\section{Results \\ 3.1. Patients}

We identified 110 consecutive patients with newly diagnosed AML undergoing ASCT in first remission after intensive induction chemotherapy. The clinical characteristics of all patients are shown in Table 1 . The median age was 54 years in both groups $(p=0.415)$, and $54.8 \%$ of patients in the $\mathrm{CH}$ group and $44.3 \%$ in the no $\mathrm{CH}$ group were female $(p=0.397)$. Favorable risk disease, according to ELN guidelines, was present in $45.2 \%$ of the patients in the $\mathrm{CH}$ group and $50.6 \%$ of those in the non- $\mathrm{CH}$ group ( $p=0.674$ ), while $48.4 \%$ versus $41.7 \%$ $(p=0.670)$ had intermediate risk AML, and $6.5 \%$ versus $7.6 \%(p>0.999)$ had adverse risk. The subgroups were equally distributed with the exception of patients with both mutated NPM1 and FLT3-ITD, which were more common in the $\mathrm{CH}$ group (19.4 vs. $5.1 \%, p=0.029)$.

Table 1. Baseline characteristics for patients included in the study. The $p$ values are for differences between the DTA-CH (clonal hematopoiesis) and no $\mathrm{CH}$ groups. IQR: interquartile range; NOS: not otherwise specified; Mut: mutated; w/o: without; ASCT: autologous hematopoietic stem cell transplantation; BW: body weight. FLT3-ITD allelic ratios were $<0.5$ (low) and $\geq 0.5$ (high).

\begin{tabular}{|c|c|c|c|c|}
\hline Parameter & $\begin{array}{c}\text { DTA-CH } \\
(n=31)\end{array}$ & $\begin{array}{l}\text { No CH } \\
(n=79)\end{array}$ & $\begin{array}{c}\text { Total } \\
(n=110)\end{array}$ & $p$ \\
\hline \multicolumn{5}{|l|}{ Demographics } \\
\hline Female sex-no. (\%) & $17(55)$ & $45(44)$ & $52(47)$ & 0.397 \\
\hline Median age-years (IQR) & $54(41-63)$ & $54(40-61)$ & $54(40-61)$ & 0.415 \\
\hline \multicolumn{5}{|l|}{ Parameters at Diagnosis, Median (Range) } \\
\hline Leukocytes (G/L) & $16.2(1.2-303)$ & $7.0(0.6-240)$ & $8.6(0.6-303)$ & 0.262 \\
\hline Neutrophils (G/L) & $1.7(0.2-50.2)$ & $1.1(0.0-19.4)$ & $1.4(0.0-50.2)$ & 0.035 \\
\hline Platelets (G/L) & $80(9-268)$ & $63(7-714)$ & $69(7-714)$ & 0.586 \\
\hline Hemoglobin $(\mathrm{g} / \mathrm{L})$ & $99(61-137)$ & $89(37-151)$ & $95(37-151)$ & 0.163 \\
\hline Peripheral blasts (\%) & $44(0-94)$ & $41(0-97)$ & $44(0-97)$ & 0.924 \\
\hline Bone marrow blasts (\%) & 75 (30-95) & $80(0-95)$ & $80(0-95)$ & 0.646 \\
\hline \multicolumn{5}{|l|}{ ELN Risk Groups-no. (\%) } \\
\hline Favorable & $14(45)$ & $40(51)$ & $54(49)$ & 0.674 \\
\hline $\mathrm{t}(8 ; 21) / R U N X 1-R U N X 1 T 1$ & $2(7)$ & $9(11)$ & $11(10)$ & 0.725 \\
\hline $\operatorname{inv}(16) / C B F B-M Y H 11$ & $2(7)$ & $8(10)$ & $10(9)$ & 0.722 \\
\hline Mut. CEBPA & $1(3)$ & $4(5)$ & $5(5)$ & $>0.999$ \\
\hline Mut. NPM1 w/o FLT3-ITD/FLT3-ITD (low allelic ratio) & $9(29)$ & $19(24)$ & $28(26)$ & 0.630 \\
\hline Intermediate & $15(48)$ & $33(42)$ & $52(43)$ & 0.670 \\
\hline Mut. NPM1 and FLT3-ITD (high allelic ratio) & $6(19)$ & $4(5)$ & $10(9)$ & 0.029 \\
\hline NOS & $9(29)$ & $29(37)$ & $38(35)$ & 0.509 \\
\hline Adverse & $2(7)$ & $6(8)$ & $8(7)$ & $>0.999$ \\
\hline Wild-type NPM1 and FLT3-ITD (high allelic ratio) & $2(7)$ & $1(1)$ & $3(3)$ & 0.191 \\
\hline Monosomal or complex karyotype & 0 & $2(3)$ & $2(2)$ & $>0.999$ \\
\hline $\mathrm{t}(\mathrm{v} ; 11) / K M T 2 A$ rearranged & 0 & $1(1)$ & $1(1)$ & $>0.999$ \\
\hline Mut. RUNX1 & 0 & $1(1)$ & $1(1)$ & $>0.999$ \\
\hline Mut. TP53 & 0 & $1(1)$ & $1(1)$ & $>0.999$ \\
\hline \multicolumn{5}{|c|}{ High-Dose Chemotherapy and Autologous Stem Cell Transplantation } \\
\hline \multicolumn{5}{|l|}{ Conditioning regimen - no. $(\%)$} \\
\hline Busulfan/Cyclophosphamide & $25(80)$ & $63(80)$ & $88(80)$ & $>0.999$ \\
\hline Busulfan/Melphalan & $4(13)$ & $9(11)$ & $13(12)$ & $>0.999$ \\
\hline Treosulfan/Melphalan & $2(7)$ & $7(9)$ & $9(8)$ & $>0.999$ \\
\hline Median time to ASCT (IQR)—months & $3.4(3.2-3.8)$ & $3.7(3.2-4.5)$ & $3.7(3.2-4.3)$ & 0.153 \\
\hline Median CD34+ transfused- $10^{6} / \mathrm{kg}$ BW & $3.81(3.1-5.2)$ & $4.20(3.3-5.2)$ & $4.17(3.2-5.2)$ & 0.448 \\
\hline
\end{tabular}




\subsection{Treatment}

The patients were treated within the previously reported HOVON/SAKK protocols. Induction treatment consisted of standard $7+3$ chemotherapy, given for two cycles. The median time from diagnosis to ASCT was 3.4 months in the $\mathrm{CH}$ group and 3.7 months in the group without $\mathrm{CH}(p=0.153)$. Conditioning for ASCT consisted of $\mathrm{BuCy}(1 \mathrm{mg} / \mathrm{kg} \mathrm{q} 6 \mathrm{~h}$ oral busulfan on days -5 to -2 for a total dose of $16 \mathrm{mg} / \mathrm{kg}$ and $60 \mathrm{mg} / \mathrm{kg}$ i.v. cyclophosphamide on days -2 and -1$)$ in $25(80.7 \%)$ and $63(79.6 \%)$ patients, BuMel $(1 \mathrm{mg} / \mathrm{kg}$ q6h oral busulfan on days $-; 5$ to -2 and $140 \mathrm{mg} / \mathrm{m}^{2}$ i.v. melphalan on days -2 and -1$)$ in $4(12.9 \%)$ and 9 (11.4\%) patients or with TreoMel $\left(14 \mathrm{mg} / \mathrm{m}^{2}\right.$ i.v. treosulfan on days -4 to -2 and $140 \mathrm{mg} / \mathrm{m}^{2}$ i.v. melphalan on day -1$)$ in $2(6.5 \%)$ and $7(8.9 \%)$ patients (Table 1). The stem cells were retransfused on day 0 . The number of re-transfused CD34+ cells was not different between patients with $\mathrm{CH}$ and without $\mathrm{CH}\left(3.8\right.$ and $4.2 \times 10^{6}$ cells $/ \mathrm{kg}$ body weight, $p=0.448)$. Of the patients with $I D H 2$ mutations, two without $\mathrm{CH}(2.5 \%)$ and one patient $(3.2 \%)$ with $\mathrm{CH}$ underwent maintenance therapy with enasidenib after ASCT, while one patient with $\mathrm{CH}$ and FLT3-ITD received midostaurin maintenance, and 8 patients with adverse risk cytogenetics and good response to induction treatment underwent autologous transplantation in the absence of an available donor or because the patients declined allogeneic transplantation.

\subsection{Clonal Hematopoiesis}

Following the definition above, $\mathrm{CH}$ of DTA after autologous transplantation was observed in 31 patients (28.2\%). The distribution of mutations at diagnosis and remission, as well as the variant allele frequencies of DTA mutations during remission, is summarized in Supplemental Figure S1. Mutations in DNMT3A were the most common and were observed in 24 patients $(77.4 \%)$, whereas TET2-CH mutation was found in 7 patients $(22.6 \%)$ and ASXL1-CH mutation was found in 4 patients (12.9\%). Meanwhile, four patients $(12.9 \%)$ had DTA-related CH mutations in more than one gene (3 DNMT3A + TET2, 1 DNMT3A + ASXL1), while two patients with CH had two mutations in the same gene (1 DNMT3A, 1 TET2).

Leukemia-related DTA mutations with molecular clearance after leukemia treatment were present in an additional 13 patients $(11.8 \%)$. Again, the distribution was similar to the patients with $\mathrm{CH}$-related mutations, with $D N M T 3 A$ being most frequent (six patients $(46.2 \%)$ ) followed by TET2 (five patients $(38.5 \%)$ ) and ASXL1 mutations (two patients $(15.4 \%))$.

\subsection{Survival Analysis}

The survival data are summarized in Figure 1 and Table 2. The median follow-up for the whole cohort was 51.3 months (93 months in the $\mathrm{CH}$ group and 46.1 months in the no $\mathrm{CH}$ group). The median progression-free survival was 26.9 months in patients without DTA mutations and 16.7 months in patients with CH-related DTA mutations (HR 0.75 $(0.42-1.33), p=0.287)$. The median overall survival was 80.9 and 54.4 months, respectively (HR $0.79(0.41-1.51), p=0.440)$. Early mortality at 100 days after ASCT was higher in the $\mathrm{CH}$ group (12.9 vs. $1.3 \%, p=0.022)$; however, this did not translate into adverse long-term survival, as the 2-year progression-free survival probability was $0.39(0.34-0.57)$ in the $\mathrm{CH}$ group and $0.46((0.22-0.56), p=0.391)$ in the no $\mathrm{CH}$ group, and the 2-year overall survival probabilities were $0.54(0.35-0.71)$ and $0.63((0.49-0.73), p=0.251)$, respectively. The relapse rate was similar between the $\mathrm{CH}$ and no $\mathrm{CH}$ group (51.6 vs. $41.7 \%, p=0.398$ ), and the number of patients undergoing allogeneic hematopoietic stem cell transplantation in CR2 did not differ significantly (19.4 vs. $24.1 \%, p=0.801)$. 


\section{Progression free survival}

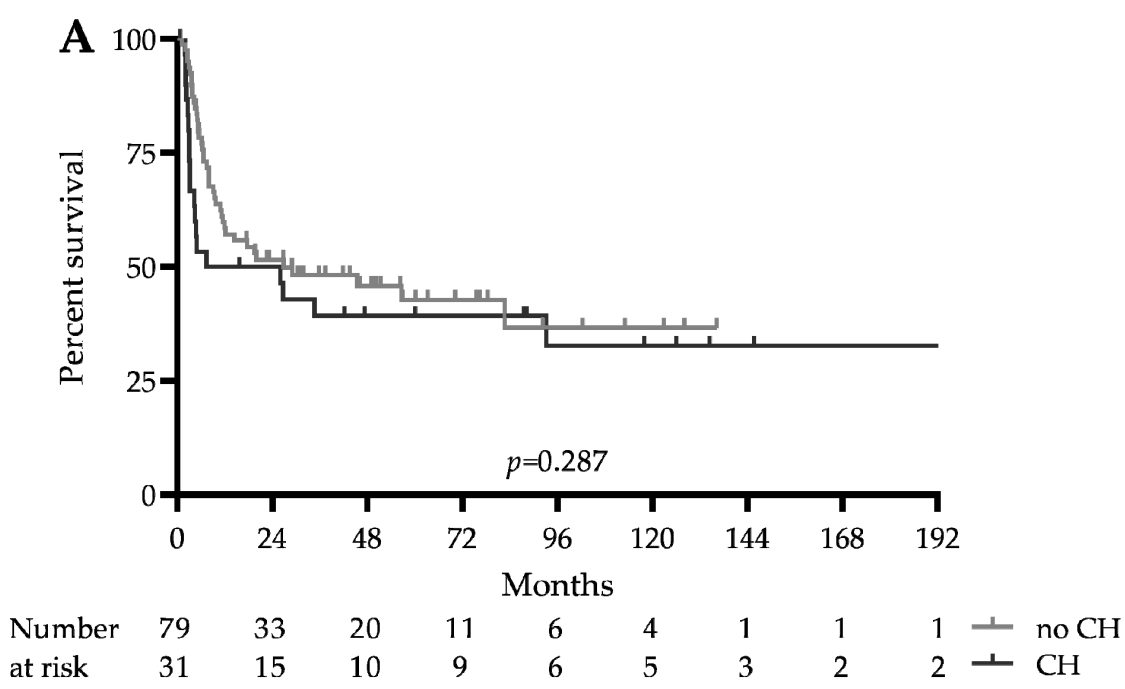

\section{Overall survival}

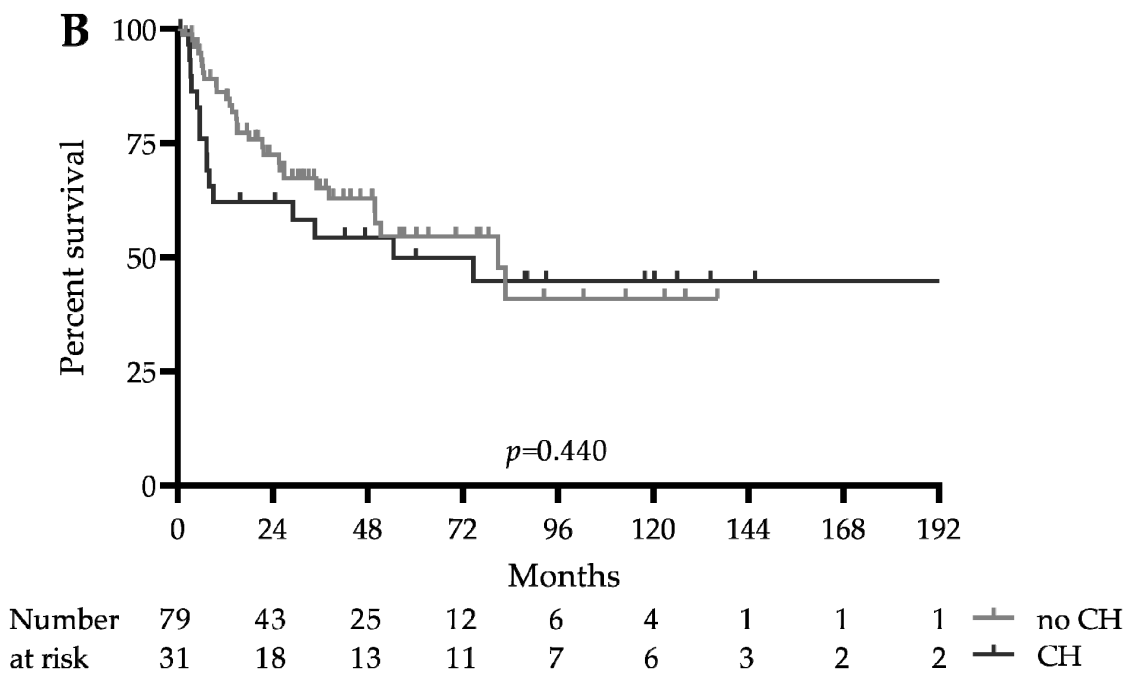

Figure 1. Survival: Kaplan-Meier survival estimates of patients with and without DTA clonal hematopoiesis (CH). (A) Progression-free survival. (B) Overall survival.

Table 2. Outcome of patients according to DTA-CH (clonal hematopoiesis) status after autologous stem cell transplantation (ASCT). CR: complete remission.

\begin{tabular}{|c|c|c|c|}
\hline Parameter & $\begin{array}{c}\mathrm{CH} \\
(n=31)\end{array}$ & $\begin{array}{l}\text { No CH } \\
(n=79)\end{array}$ & $p$ \\
\hline \multicolumn{4}{|l|}{ Survival } \\
\hline Median follow up for OS-months & 93.0 & 46.1 & - \\
\hline Median progression free survival—months & 16.7 & 26.9 & 0.287 \\
\hline Median overall survival—months & 54.4 & 80.9 & 0.440 \\
\hline 2-year progression free survival probability $(95 \% \mathrm{CI})$ & $0.39(0.34-0.57)$ & $0.46(0.22-0.56)$ & 0.391 \\
\hline 2-year overall survival probability (95\%CI) & $0.54(0.35-0.71)$ & $0.63(0.49-0.73)$ & 0.251 \\
\hline 30-day mortality-no. (\%) & 0 & 0 & $>0.999$ \\
\hline Allogeneic transplantation in CR2-no. (\%) & $6(19)$ & $19(24)$ & 0.801 \\
\hline
\end{tabular}




\subsection{Survival of DTA Subgroups}

Survival analysis of the DTA subgroups is summarized in Supplemental Figure S2. When analyzing the DNMT3A, TET2, and ASXL1 CH-mutations individually, patients with persistent mutations in TET2 and DNMT3A mutations had similar survival outcomes to patients without $\mathrm{CH}$, while patients with persistent ASXL1 mutations showed a nonsignificant trend toward better outcomes than patients without $\mathrm{CH}$ in the respective gene. However, the small number of patients in the TET2 and ASXL1 subgroups did not warrant a conclusive statement.

\subsection{Secondary Malignancies}

Three patients-two in the no $\mathrm{CH}$ group and one in the $\mathrm{CH}$ group-developed a second malignancy after ASCT. One patient was diagnosed with extensive disease smallcell lung cancer 6 years after ASCT, and one was diagnosed with metastatic adenocarcinoma of a gastrointestinal origin 7 years after ASCT. Both patients succumbed to these diseases. Most interestingly, one patient who underwent ASCT for AML with mutated NPM1 and FLT3-ITD and who had no DTA-CH was diagnosed with AML with an IDH2 mutation 8.5 years later without evidence of either mutated NMP1 or FLT3-ITD or DTA mutations.

\subsection{Different VAF Cutoffs}

For the principal analysis, we applied a VAF cutoff for the definition of $\mathrm{CH}$ of $2 \%$ [8]. We also investigated the impact on the survival rates by applying $5 \%$ and $10 \%$ VAF cutoffs for the $\mathrm{CH}$ DTA mutations. Elevation of the VAF cutoff had no significant impact on survival, as summarized in Supplemental Figure S3.

\section{Discussion}

The increasing availability of NGS has led to the discovery of a multitude of novel mutations of, in part, indeterminate significance in AML, as well as other malignancies $[9,17]$. The prevalence of $\mathrm{CH}$-related DTA mutations is known to increase with age, and patients with $\mathrm{CH}$ have an increased risk of developing hematologic malignancies, cardiovascular disorders and death [8]. Previous investigations found evidence of $\mathrm{CH}$ in between $65 \%$ and $75 \%$ of patients with AML [18-20]. This rate is higher than that in our cohort; however, the mentioned studies investigated more genes and used VAF cutoffs between $0.5 \%$ and $1 \%$.

While the impact of $\mathrm{CH}$ on the risk of disease development has been intensively studied, less data are available on the impact of DTA mutations during complete remission and, to the best of our knowledge, no previous study has investigated the prognostic impact of $\mathrm{CH}$ for patients undergoing HDCT or ASCT for AML in CR1.

Autologous stem cell transplantation is one possibility for consolidation treatment for patients with good and intermediate risk AML in first complete remission. In ASCT, cells are harvested after induction and reinfused after high-dose chemotherapy consolidation to shorten marrow aplasia and reduce the risk for infectious complications. Harvesting stem cells from patients with hematologic malignancies poses a potential risk for the retransfusion of mutant cells. Retransfusion of DTA mutant cells may therefore pose a risk for development of a hematologic malignancy, AML relapse, or death.

First, we observed $\mathrm{CH}$ for DTA mutations at a frequency of $28.2 \%$ in our cohort during complete remission, which was similar to previous analyses in AML patients. Jongen-Lavrencic et al. reported a DTA-CH rate of $23 \%$ during complete remission, while Grimm et al. reported $\mathrm{CH}$-related DTA mutations to be present at a rate of $35.4 \%$ during $\mathrm{CR}[4,21]$. The detected rate of $\mathrm{CH}$ was also similar to that reported by Mouhieddine et al. in patients with multiple myeloma (21.6\%) [22] or by Gibson et al. in patients with lymphoma (29.9\%) [23] prior to ASCT. However, these analyses included small numbers of other CH-related mutations such as TP53 or PPM1D.

Secondly, our analysis found no evidence for more adverse progression-free or overall survival outcomes in patients with persistent DTA-CH after HDCT or ASCT compared with patients without this phenomenon. These results are in line with a previous study by 
Jongen-Lavrencic et al. that showed no correlation between the detection of CH-related DTA mutations during complete remission and adverse outcomes in AML patients [4]. This analysis included a limited number of 78 patients $(18 \%)$ that underwent ASCT, whereas almost half of the patients (44\%) underwent allogeneic transplantation as a consolidation treatment. In comparison, our study cohort consisted of a homogenous and rather large sample of 110 mostly good and intermediate risk patients undergoing HDCT or ASCT as a consolidation in first remission.

Early mortality in the first 100 days was higher in the no $\mathrm{CH}$ group, but the reason for this finding remains unclear and, importantly, this did not translate into adverse long-term outcomes. Larger cohorts are needed to exclude any by chance findings. Similarly, the number of patients dying due to non-AML related reasons remained too small to allow or support conclusions on the development of or death from, for example, vascular disease.

In light of our results, the presence of DTA-CH in AML patients after HDCT or ASCT does not warrant intensification of treatment by allogeneic transplantation. Moreover, the presence of DTA-CH should not prevent eligible patients from undergoing autologous transplantation in first complete remission. While there was a tendency toward more adverse survival outcomes in patients with DTA mutations at VAFs over $10 \%$, this did not reach statistical significance. Still, these patients may reflect a subset prone to relapse and death which may benefit from intensified surveillance and early intervention in the case of indication of a relapse.

In 2020, Gibson et al. investigated whether $\mathrm{CH}$ affecting allogeneic donors had an impact on the outcomes of 1727 patients receiving allogeneic HSCT from donors aged 40 years and older due to myeloid $(53.8 \%)$ or lymphatic malignancies $(41.6 \%)$. CH was present in a relevant proportion of $22.5 \%$ of donor samples. Surprisingly, evidence of donor DNMT3A-CH was associated with improved PFS and OS in the allo-transplant recipients, while other CH-related mutations (TET2, ASXL1, and PPM1D) had no impact on the outcomes. While the exact mechanism is unclear, it is suggested that DNMT3A mutations lead to improved T-cell immune activity, which is a key determinant of transplant activity. This is also supported by the observation that in patients who received post-transplant cyclophosphamide for GvHD prophylaxis, no impact due to the DNMT3A status on the survival outcome was observed. In addition, $\mathrm{CH}$ involving other gene mutations had no impact on the outcome [24]. Shlush et al. demonstrated that DNMT3A mutations can also be present in non-myeloid cells [25]. In our analysis, we found no difference in outcomes in the DNMT3A-CH subgroup compared with patients without $\mathrm{CH}$ in the respective gene. However, there were major differences in the patient population as well as treatment between our study and the mentioned study. In contrast, two investigations found the presence of CH involving DNMT3A, TET2, ASXL1, TP53, and PPM1D among others to be associated with inferior outcomes in patients undergoing autologous hematopoietic transplantation for multiple myeloma [22] and lymphoma [23]. Thus, possible differences in the long-term outcomes should be discussed in patients with $\mathrm{CH}$ receiving ASCT and in patients receiving allogeneic HSCT from donors with $\mathrm{CH}$ for different indications or different hematologic entities, respectively.

\section{Conclusions}

In conclusion, our study found no evidence of a worse prognosis for AML patients with DTA CH undergoing HDCT or ASCT in CR1. These data have a clinical impact, as they give no evidence for excluding AML patients with evidence of DTA CH from ASCT concepts. Our data should be independently confirmed in adequately powered prospective future studies. Due to the relatively small rate of progression from $\mathrm{CH}$ to overt hematologic malignancy $(0.5-1 \%$ per year) [8,10], analyses from large registries with a sufficiently long follow-up will be needed to ultimately answer the issues raised in this study. 
Supplementary Materials: The following are available online at https:/ / www.mdpi.com/article / 10.3390/cancers13133190/s1, Figure S1: Distribution of mutations and variant allele frequencies (VAFs); Figure S2: Survival according to subgroups of clonal hematopoiesis (CH); Figure S3: Survival according to different VAF (variant allele frequencies) cutoffs.

Author Contributions: Conceptualization, T.P.; methodology, T.P., and N.P. and A.D.H.; data curation, A.D.H. and N.P.; writing — original draft preparation, A.D.H.; writing-review and editing, all authors. All authors have read and agreed to the published version of the manuscript.

Funding: This research received no external funding.

Institutional Review Board Statement: This study was approved by a decision of the local ethics committee in Berne, Switzerland.

Informed Consent Statement: Informed consent was obtained from all subjects involved in the study.

Data Availability Statement: The data generated during and/or analyzed during this study are available from the corresponding author on reasonable request.

Conflicts of Interest: The authors declare no conflict of interest.

\section{References}

1. Döhner, H.; Estey, E.; Grimwade, D.; Amadori, S.; Appelbaum, F.R.; Büchner, T.; Dombret, H.; Ebert, B.L.; Fenaux, P.; Larson, R.A.; et al. Diagnosis and management of AML in adults: 2017 ELN recommendations from an international expert panel. Blood 2017, 129, 424-447. [CrossRef]

2. Wetzel, D.; Mueller, B.U.; Mansouri Taleghani, B.; Baerlocher, G.M.; Seipel, K.; Leibundgut, K.; Pabst, T. Delayed Haematological recovery after autologous stem cell transplantation is associated with favourable outcome in acute myeloid leukaemia. $\mathrm{Br}$. $J$. Haematol. 2015, 168, 268-273. [CrossRef] [PubMed]

3. de Benito, A.S.; Jeker, B.; Gfeller, E.; Porret, N.; Banz, Y.; Novak, U.; Bacher, U.; Pabst, T. Molecular minimal residual disease negativity and decreased stem cell mobilization potential predict excellent outcome after autologous transplant in NPM1 mutant acute myeloid leukemia. Haematologica 2020, 105, e9-e12. [CrossRef] [PubMed]

4. Jongen-Lavrencic, M.; Grob, T.; Hanekamp, D.; Kavelaars, F.G.; Al Hinai, A.; Zeilemaker, A.; Erpelinck-Verschueren, C.A.J.; Gradowska, P.L.; Meijer, R.; Cloos, J.; et al. Molecular Minimal Residual Disease in Acute Myeloid Leukemia. N. Engl. J. Med. 2018, 378, 1189-1199. [CrossRef]

5. Flach, J.; Shumilov, E.; Joncourt, R.; Porret, N.; Tchinda, J.; Legros, M.; Scarpelli, I.; Hewer, E.; Novak, U.; Schoumans, J.; et al. Detection of rare reciprocal RUNX1 rearrangements by next-generation sequencing in acute myeloid leukemia. Genes Chromosom. Cancer 2020, 59, 268-274. [CrossRef] [PubMed]

6. Flach, J.; Shumilov, E.; Wiedemann, G.; Porret, N.; Shakhanova, I.; Bürki, S.; Legros, M.; Joncourt, R.; Pabst, T.; Bacher, U. Clinical potential of introducing next-generation sequencing in patients at relapse of acute myeloid leukemia. Hematol. Oncol. 2020, 38, 425-431. [CrossRef] [PubMed]

7. Shumilov, E.; Flach, J.; Kohlmann, A.; Banz, Y.; Bonadies, N.; Fiedler, M.; Pabst, T.; Bacher, U. Current status and trends in the diagnostics of AML and MDS. Blood Rev. 2018, 32, 508-519. [CrossRef] [PubMed]

8. Shlush, L.I. Age-related clonal hematopoiesis. Blood 2018, 131, 496-504. [CrossRef]

9. Bacher, U.; Shumilov, E.; Flach, J.; Porret, N.; Joncourt, R.; Wiedemann, G.; Fiedler, M.; Novak, U.; Amstutz, U.; Pabst, T. Challenges in the introduction of next-generation sequencing (NGS) for diagnostics of myeloid malignancies into clinical routine use. Blood Cancer J. 2018, 8, 113. [CrossRef]

10. Steensma, D.P.; Bejar, R.; Jaiswal, S.; Lindsley, R.C.; Sekeres, M.A.; Hasserjian, R.P.; Ebert, B.L. Clonal hematopoiesis of indeterminate potential and its distinction from myelodysplastic syndromes. Blood 2015, 126, 9-16. [CrossRef]

11. Genovese, G.; Kähler, A.K.; Handsaker, R.E.; Lindberg, J.; Rose, S.A.; Bakhoum, S.F.; Chambert, K.; Mick, E.; Neale, B.M.; Fromer, M. Clonal hematopoiesis and blood-cancer risk inferred from blood DNA sequence. N. Engl. J. Med. 2014, 371, 2477-2487. [CrossRef] [PubMed]

12. Jaiswal, S.; Fontaninallas, P.; Flannick, J.; Manning, A.; Grauman, P.V.; Mar, B.G.; Lindsley, R.C.; Mermel, C.H.; Burtt, N.; Chavez, A.; et al. Age-related clonal hematopoiesis associated with adverse outcomes. N. Engl. J. Med. 2014, 371, 2488-2498. [CrossRef] [PubMed]

13. Mueller, B.U.; Seipel, K.; Bacher, U.; Pabst, T. Autologous transplantation for older adults with AML. Cancers 2018, 10, 340. [CrossRef]

14. Sula, M.; Bacher, U.; Oppliger-Leibundgut, E.; Mansouri Taleghani, B.; Novak, U.; Pabst, T. Excellent outcome after consolidation with autologous transplantation in patients with core binding factor acute myeloid leukemia. Bone Marrow Transplant. 2020, 55, 1690-1693. [CrossRef]

15. von Grünigen, I.; Raschle, J.; Rüsges-Wolter, I.; Taleghani Behrouz, M.; Mueller, B.U.; Pabst, T. The relapse risk of AML patients undergoing autologous transplantation correlates with the stem cell mobilizing potential. Leuk. Res. 2012, 36, 1325-1329. [CrossRef] 
16. Cheson, B.D.; Bennett, J.M.; Kopecky, K.J.; Büchner, T.; Willman, C.L.; Estey, E.H.; Schiffer, C.A.; Doehner, H.; Tallman, M.S.; Lister, T.A.; et al. Revised Recommendations of the International Working Group for diagnosis, standardization of response criteria, treatment outcomes, and reporting standards for therapeutic trials in acute myeloid leukemia. J. Clin. Oncol. 2003, 21, 4642-4649. [CrossRef] [PubMed]

17. Serratì, S.; de Summa, S.; Pilato, B.; Petriella, D.; Lacalamita, R.; Tommasi, S.; Pinto, R. Next-generation sequencing: Advances and applications in cancer diagnosis. Onco Targets. Ther. 2016, 9, 7355-7365. [CrossRef]

18. Young, A.L.; Spencer Tong, R.; Birmann, B.M.; Druley, T.E. Clonal hematopoiesis and risk of acute myeloid leukemia. Haematologica 2019, 104, 2410-2417. [CrossRef]

19. Abelson, S.; Collord, G.; Ng, S.W.K.; Weissbrod, O.; Mendelson Cohen, N.; Niemeyer, E.; Barda, N.; Zuzarte, P.C.; Heisler, L.; Sundaravadanam, Y.; et al. Prediction of acute myeloid leukaemia risk in healthy individuals. Nature 2018, 559, 400-404. [CrossRef]

20. Desai, P.; Mencia-Trinchant, N.; Savenkov, O.; Simon, M.S.; Cheang, G.; Lee, S.; Samuel, M.; Ritchie, E.K.; Guzman, M.L.; Ballman, K.V.; et al. Somatic mutations precede acute myeloid leukemia years before diagnosis. Nat. Med. 2018, 24, 1015-1023. [CrossRef]

21. Grimm, J.; Bill, M.; Jentzsch, M.; Beinicke, S.; Häntschel, J.; Goldmann, K.; Schulz, J.; Cross, M.; Franke, G.N.; Behre, G.; et al. Clinical impact of clonal hematopoiesis in acute myeloid leukemia patients receiving allogeneic transplantation. Bone Marrow Transplant. 2019, 54, 1189-1197. [CrossRef]

22. Mouhieddine, T.H.; Sperling, A.S.; Redd, R.; Park, J.; Leventhal, M.; Gibson, C.J.; Manier, S.; Nassar, A.H.; Capelletti, M.; Huynh, D.; et al. Clonal hematopoiesis is associated with adverse outcomes in multiple myeloma patients undergoing transplant. Nat. Commun. 2020, 11, 2996. [CrossRef] [PubMed]

23. Gibson, C.J.; Lindsley, R.C.; Tchekmedyian, V.; Mar, B.G.; Shi, J.; Jaiswal, S.; Bosworth, A.; Francisco, L.; He, J.; Bansal, A.; et al. Clonal hematopoiesis associated with adverse outcomes after autologous stem-cell transplantation for lymphoma. J. Clin. Oncol. 2017, 35, 1598-1605. [CrossRef] [PubMed]

24. Gibson, C.J.; Kim, H.T.; Murdock, H.M.; Hambley, B.; Zhao, L.; Green, L.; Fleharty, M.; Blumenstiel, B.; Cibulskis, C.; Gocke, C.D.; et al. DNMT3A clonal hematopoiesis in older donors is associated with improved survival in recipients after allogeneic hematopoietic cell transplant. Blood 2020, 136, 26. [CrossRef]

25. Shlush, L.I.; Zandi, S.; Mitchell, A.; Chen, W.C.; Brandwein, J.M.; Gupta, V.; Kennedy, J.A.; Schimmer, A.D.; Schuh, A.C.; Yee, K.W.; et al. Identification of pre-leukaemic haematopoietic stem cells in acute leukaemia. Nature 2014, 506, 328-333. [CrossRef] [PubMed] 